\title{
Half-integer Higher Spin Fields in (A)dS from Spinning Particle Models
}

\author{
Olindo Corradini \\ Dipartimento di Fisica, Università di Bologna and \\ INFN, Sezione di Bologna, via Irnerio 46, I-40126 Bologna, Italy \\ E3 \\ Centro de Estudios en Física y Matemáticas Basicas y Aplicadas \\ Universidad Autónoma de Chiapas, Tuxtla Gutiérrez, Chiapas, Mexico \\ E-mail: corradini@bo.infn.it
}

\begin{abstract}
We make use of $O(2 r+1)$ spinning particle models to construct linearized higher-spin curvatures in (A)dS spaces for fields of arbitrary half-integer spin propagating in a space of arbitrary (even) dimension: the field potentials, whose curvatures are computed with the present models, are spinor-tensors of mixed symmetry corresponding to Young tableaux with $\frac{D}{2}-1$ rows and r columns, thus reducing to totally symmetric spinor-tensors in four dimensions. The paper generalizes similar results obtained in the context of integer spins in (A)dS.
\end{abstract}




\section{Contents}

1. Introduction 1

2. Constraint algebra in flat space and (A)dS space 3

3. Higher spin equations of motion in flat space 6

4. Higher spin equations of motion in (A)dS space 10

5. Conclusions and Outlook 14

\section{Introduction}

Despite years of investigation higher-spin field theory (see [1] for a review) is still not a completely understood subject. In particular the introduction of interactions has turned out to be a formidable task. Thus, in order to clarify such difficult problem it may be fruitful to investigate the subject from different perspectives such as the first-quantized approach offered by some spinning particle models.

Particle models constitute an alternative to conventional (second-quantized) field theories in the study of quantum field theory by means of the worldline formalism [2] and can also be applied in the study of higher-spins field theory; in particular $O(N)$ extended spinning particle models $[3,4]$ turn out to be related to the geometric formulation of higher spin field theory. In the context of second-quantized field theory, geometric equations of motions for totally symmetric higher spin fields were proposed in [5] (using linearized higher spin geometry constructed in [6], further developed in [7] and generalized to mixed symmetry tensors in [8]) in order to relax the algebraic constraints present in the conventional formulation of higher spin equations of motion in terms of Lorentz-covariant second-order (first-order for half integer spins) differential equation of the field potential. For integer spins, lagrangians for totally symmetric tensors in flat space and (A)dS space were first constructed by Fronsdal [9] and later generalized to mixed symmetry tensors in [10], whereas for half-integer fields, lagrangians for totally symmetric (spinor)-tensor fields in flat space were first proposed by Fang and Fronsdal [11] and Curtright [12], whereas extensions to (A)dS spaces were soon later obtained in [13]. (Fang)-Fronsdal equations of motion are characterized by (gamma)-trace constraints which must be satisfied by fields 
and gauge parameters and can be obtained by partial gauging of the unconstrained (compensated) geometric equations of motion.

Geometric equations of motion are a natural outcome of spinning particles: the canonical quantization of locally-supersymmetric $O(N)$-extended spinning particle models

$$
\begin{gathered}
S=\int d t[p_{\mu} \dot{x}^{\mu}+\frac{i}{2} \psi_{i \mu} \dot{\psi}_{i}^{\mu}-e \underbrace{\left(\frac{1}{2} p_{\mu} p^{\mu}\right)}_{H}-i \chi_{i} \underbrace{\left(p_{\mu} \psi_{i}^{\mu}\right)}_{Q_{i}}-\frac{i}{2} a_{i j} \underbrace{\left(\psi_{i}^{\mu} \psi_{j \mu}\right)}_{J_{i j}}] \\
\quad i=1, \ldots, N
\end{gathered}
$$

yields equations of motions for spin- $\frac{N}{2}$ fields (wave functions) in terms of the corresponding linearized curvatures. For flat external backgrounds the correspondence is well established (see [14] for a recent very detailed analysis) and one-loop path integral results were also obtained [15]. The coupling to external arbitrary backgrounds is possible for $N \leq 2$, corresponding to worldline descriptions of spin $\leq 1$ particles coupled to gravity [16]. However, for $N>2$, the coupling of the above models to external arbitrary backgrounds is problematic [4], reflecting the aforementioned difficulty of introducing interactions in higher spin fields. A partial way-out to such problem was given in [17] where (using the manifestly conformally-invariant formulation of $[18,19])$ it was shown how to consistently couple $O(N)$ spinning particles to AdS backgrounds. In [20] spinning particle models with $O(N)$-extended local supersymmetry were further analyzed and coupling to a more generic set of backgrounds, conformally flat spaces, was proposed: linearized higher-spin curvatures and geometric equations of motion of integer higher-spin fields (with $N=2 s, s \in \mathbb{N}$ ) in (A)dS spaces were derived and studied, from the canonical quantization of the models (higher-spin de Wit-Freedman linearized curvatures for totally symmetric potentials in AdS were constructed in [21].)

The present manuscript is a generalization of the results obtained in [20] to halfinteger higher-spin fields, in (A)dS. Here we restrict our study to the link between $O(N)$ spinning particles and fermionic higher spin fields. However, it is worth mentioning other particle models relevant to higher spin physics, such as twistor-like superparticles [22], $U(N)$ spinning particles [23] and $S p(2 r)$ models [24] (obtained from gauging orthosymplectic models [25]) whose BRST detour quantization [26] describes higher spins of mixed symmetry type [27, 8]. The structure of the paper is as follows: in Section 2 we describe the quantum constraint algebra associated to the flat space particle action (1.1) and its quadratically-deformed (A)dS version (quadratic algebras have already appeared in the study of higher spin fields [28]). The geometric equations of motion for fermionic higher spin fields in flat space and (A)dS space are obtained in Section 3 and Section 4 respectively, by imposing the constraint algebra onto the physical wave function, hence establishing a dictionary between the present particle formulation and the conventional second-quantized for- 
mulation. In Section 5 we provide some conclusions and future investigations that may be undertaken within the present approach.

\section{Constraint algebra in flat space and (A)dS space}

In order to obtain the quantum $O(N)$-extended supersymmetric particle algebra (with $N=2 r+1$ ) in flat space, from the classical constraints of (1.1), one only needs to specify the correct ordering in the definition of the $S O(N)$ generators $J_{i j}$, as there are no ordering ambiguities associated with the hamiltonian $H$ and susy generators $Q_{i}$. Taking that into account, the quantum constraints are given by

$$
H=\frac{1}{2} p_{\mu} p^{\mu}, \quad Q_{i}=p_{\mu} \psi_{i}^{\mu}, \quad J_{i j}=\frac{1}{2}\left[\psi_{i}^{\mu}, \psi_{j \mu}\right]
$$

with $\left\{\psi_{i}^{\mu}, \psi_{j}^{\nu}\right\}=\eta^{\mu \nu} \delta_{i j}$. They satisfy the quantum algebra

$$
\begin{aligned}
{\left[J_{i j}, J_{k l}\right] } & =-\delta_{j k} J_{i l}+\delta_{i k} J_{j l}+\delta_{j l} J_{i k}-\delta_{i l} J_{j k} \\
{\left[J_{i j}, Q_{k}\right] } & =\delta_{j k} Q_{i}-\delta_{i k} Q_{j} \\
\left\{Q_{i}, Q_{j}\right\} & =2 \delta_{i j} H
\end{aligned}
$$

which is first class. The above (multi)-Clifford algebra can be realized using gamma matrices as follows [4]

$$
\psi_{1}^{\mu}=\frac{1}{\sqrt{2}} \gamma^{\mu} \otimes \gamma \cdots \otimes \gamma, \quad \psi_{2}^{\mu}=\frac{1}{\sqrt{2}} \rrbracket \otimes \gamma^{\mu} \otimes \gamma \cdots \otimes \gamma, \quad \psi_{N}^{\mu}=\frac{1}{\sqrt{2}} \llbracket \cdots \otimes \square \otimes \gamma^{\mu}
$$

with the wave function written as a multispinor $\Psi_{\alpha_{1} \cdots \alpha_{N}}$ and with $\gamma$ being the chirality matrix; throughout the paper we work with a generic even dimension $D=2 d$. In fact for $D=2 d+1, N>2$ the present models are empty due to a global anomaly [4]. In $D=4$, the above constraint algebra yields Bargmann-Wigner equations [29] for a spin- $\frac{N}{2}$ field (BRST quantization of the models is described in [30]).

Here, in order to study the spin- $s(s=N / 2=r+1 / 2)$ equations of motion, we use a different basis taking complex combinations of the first $2 r$ indices of $S O(N)$ and define (for $I, i=1, \ldots, r$ )

$$
\psi_{I}^{\mu}=\frac{1}{\sqrt{2}}\left(\psi_{i}^{\mu}+i \psi_{i+r}^{\mu}\right), \quad \bar{\psi}_{\bar{I}}^{\mu}=\frac{1}{\sqrt{2}}\left(\psi_{i}^{\mu}-i \psi_{i+r}^{\mu}\right) \equiv \psi^{I \mu}
$$

and

$$
\psi_{2 r+1}^{\mu} \equiv \frac{1}{\sqrt{2}} \gamma^{\mu}
$$

More specifically, in order to set the antisymmetry between (2.5) and (2.6) we define them as

$$
\psi_{I}^{\mu} \rightarrow \psi_{I}^{\mu} \otimes \gamma, \quad \psi^{I \mu} \rightarrow \psi^{I \mu} \otimes \gamma, \quad \gamma^{\mu} \rightarrow \square \otimes \gamma^{\mu}
$$


In the "coordinate" representation one can realize $\psi_{I}^{\mu}$ as multiplication by Grassmann variables and $\psi_{\mu}^{I}=\frac{\partial}{\partial \psi_{I}^{\mu}}$ (we use left derivatives). This realization keeps manifest only the $U(r) \subset S O(2 r+1)$ subgroup of the internal symmetry group, but will be quite useful in classifying the constraints and their solutions. In this representation the left space in (2.7) is the space of antisymmetric multiforms $\psi_{1}^{\mu_{1}} \cdots \psi_{1}^{\mu_{A_{1}}} \cdots \psi_{r}^{\nu_{1}} \cdots \psi_{r}^{\nu_{A_{r}}}$, whereas the right space is the fermionic space where the $(2 r+1)$-th Clifford algebra is realized as gamma-matrices. In terms of those operators we have

$$
\begin{aligned}
\left\{\psi_{I}^{\mu}, \psi^{J \nu}\right\} & =\eta^{\mu \nu} \delta_{I}^{J} \\
\left\{\gamma^{\mu}, \gamma^{\nu}\right\} & =2 \eta^{\mu \nu} .
\end{aligned}
$$

Henceforth we can avoid to explicitly write down the tensor product as its only effect is the aforementioned antisymmetry; we will only need to take care of imposing it.

The susy charges in the $U(r)$ basis take the form

$$
Q_{I}=\psi_{I}^{\mu} p_{\mu}, \quad Q^{I}=\psi^{I \mu} p_{\mu}
$$

and

$$
\not P=\gamma^{\mu} p_{\mu}=\sqrt{2} Q_{N}
$$

is nothing but the Dirac operator. Hence, the non-vanishing part of the susy algebra (2.4) reads

$$
\left\{Q_{I}, Q^{J}\right\}=2 \delta_{I}^{J} H, \quad P^{2}=2 H
$$

In the complex basis defined above, the $S O(N)$ generators split as $J_{i j} \sim\left(J_{I \bar{J}}, J_{I J}, J_{\bar{I} \bar{J}}, J_{N I}, J_{N \bar{I}}\right) \sim\left(J_{I}{ }^{J}, K_{I J}, K^{I J}, L_{I}, L^{I}\right)$, which we normalize as

$$
\begin{aligned}
& J_{I}^{J}=\psi_{I} \cdot \psi^{J}-d \delta_{I}^{J}, \quad K_{I J}=\psi_{I} \cdot \psi_{J}, \quad K^{I J}=\psi^{I} \cdot \psi^{J} \\
& L_{I}=\gamma^{\mu} \psi_{I \mu}, \quad L^{I}=\gamma^{\mu} \psi_{\mu}^{I}
\end{aligned}
$$

so that when $I=J, J_{I}^{J}$ is a hermitian operator with real eigenvalues; the above generators can be written in terms of the Clifford basis by means of (2.5): for example $L_{I}=\gamma^{\mu} \psi_{I \mu}=J_{N i}+i J_{N i+r}$. The $S O(N)$ algebra (2.2) breaks up into the $S O(2 r)$ subalgebra generated by (2.13) [20]. The remaining non-vanishing relations of the $S O(N)$ algebra, involving $L$ 's are

$$
\begin{aligned}
{\left[J_{I}^{J}, L_{K}\right] } & =\delta_{K}^{J} L_{I}, \quad\left[J_{I}^{J}, L^{K}\right]=-\delta_{I}^{K} L^{J} \\
{\left[K^{I J}, L_{K}\right] } & =\delta_{K}^{J} L^{I}-\delta_{K}^{I} L^{J}, \quad\left[K_{I J}, L^{K}\right]=\delta_{J}^{K} L_{I}-\delta_{I}^{K} L_{J} \\
{\left[L_{I}, L^{J}\right] } & =-2 J_{I}^{J}, \quad\left[L^{I}, L^{J}\right]=-2 K^{I J}, \quad\left[L_{I}, L_{J}\right]=-2 K_{I J} .
\end{aligned}
$$


Finally, it is useful to list in the same basis the remaining part of the constraint algebra corresponding to eq. (2.3)

$$
\begin{aligned}
& {\left[J_{I}^{J}, Q_{K}\right]=\delta_{K}^{J} Q_{I}, \quad\left[J_{I}^{J}, Q^{K}\right]=-\delta_{I}^{K} Q^{J}} \\
& {\left[K^{I J}, Q_{K}\right]=\delta_{K}^{J} Q^{I}-\delta_{K}^{I} Q^{J}, \quad\left[K_{I J}, Q^{K}\right]=\delta_{J}^{K} Q_{I}-\delta_{I}^{K} Q_{J}} \\
& {\left[L^{I}, Q_{J}\right]=\delta_{J}^{I} \mathbb{P}, \quad\left[L_{I}, Q^{J}\right]=\delta_{I}^{J} \not{P}} \\
& {\left[L_{I}, \not P\right]=-2 Q_{I}, \quad\left[L^{I}, \not P\right]=-2 Q^{I} .}
\end{aligned}
$$

As explicitly worked out in [20], the deformation of the above flat algebra to (A)dS spaces $R_{a b c d}=b\left(\eta_{a c} \eta_{b d}-\eta_{a d} \eta_{b c}\right)$, is obtained by generalizing the constraints to

$$
\begin{aligned}
& H=\frac{1}{2}\left(\pi^{a} \pi_{a}-i \omega_{a b}^{a} \pi^{b}\right)+\frac{b}{4} J_{i j} J_{i j}-b A(D), \\
& Q_{i}=\psi_{i}^{a} \pi_{a}=\psi_{i}^{a} e_{a}^{\mu}\left(p_{\mu}-\frac{1}{2} \omega_{\mu b c} M^{b c}\right), \quad J_{i j}=\frac{1}{2}\left[\psi_{i}^{a}, \psi_{j a}\right]
\end{aligned}
$$

with $M^{a b}=\frac{i}{2}\left[\psi_{i}^{a}, \psi_{i}^{b}\right]$ being the multispinor representation of the $S O(D)$ Lorentz generators, $A(D)=(2-N) \frac{D}{8}-\frac{D^{2}}{8}$ and

$$
\pi_{\mu}=p_{\mu}-\frac{1}{2} \omega_{\mu b c} M^{b c}, \quad \pi_{a}=e_{a}^{\mu} \pi_{\mu}
$$

the covariant momentum. With these operators equations (2.2-2.3) hold unchanged, whereas the susy algebra (2.4) gets modified as

$$
\left\{Q_{i}, Q_{j}\right\}=2 \delta_{i j} H+\frac{b}{2}\left(J_{i k} J_{j k}+J_{j k} J_{i k}-\delta_{i j} J_{k l} J_{k l}\right) .
$$

In the above complex basis we have $Q_{I}=\psi_{I}^{a} \pi_{a}, Q^{I}=\psi^{I a} \pi_{a}$ and $\not I=\gamma^{a} \pi_{a}$ and the susy algebra in the same basis can be obtained from (2.24) in a simple way by noting that the complexification in the internal indices (cfr. eq. (2.5)) implies the transformation of the flat metric as $\delta_{i j} \rightarrow\left(\delta_{I}{ }^{J}, \delta^{I}{ }_{J}, \delta_{N N}\right)$. Hence,

$$
\begin{aligned}
\left\{Q_{I}, Q_{J}\right\}= & b\left(K_{I L} J_{J}^{L}+K_{J L} J_{I}^{L}\right)+\frac{b}{4}\left(L_{I} L_{J}+L_{J} L_{I}\right) \\
\left\{Q_{I}, \not I\right\}= & b\left(K_{I K} L^{K}+L_{K} J_{I}^{K}+\frac{1}{2} L_{I}\right) \\
\not I^{2}= & 2\left(H_{0}-b A(D)\right)+\frac{b}{2}\left(L_{K} L^{K}+L^{K} L_{K}\right) \\
\left\{Q^{I}, Q^{J}\right\}= & b\left(-K^{I L} J_{L}^{J}-K^{J L} J_{L}^{J}\right)+\frac{b}{4}\left(L^{I} L^{J}+L^{J} L^{I}\right) \\
\left\{Q_{I}, Q^{J}\right\}= & 2 \delta_{I}^{J}\left(H_{0}-b A(D)\right)+\frac{b}{4}\left(L_{I} L^{J}+L^{J} L_{I}\right) \\
& -\frac{b}{2}\left(J_{I}^{K} J_{K}^{J}+J_{K}^{J} J_{I}^{K}-K_{I K} K^{J K}-K^{J K} K_{I K}\right) \\
\left\{Q^{I}, \not I\right\}= & b\left(-L^{K} J_{K}^{I}+K^{I K} L_{K}+\frac{1}{2} L^{I}\right) .
\end{aligned}
$$




\section{Higher spin equations of motion in flat space}

The equations of motion for the higher spin wave function are obtained by imposing that the above operators (constraints) annihilate the first-quantized physical state. A generic state in the Hilbert space where such operators act can be written as

$$
|R\rangle \sim a_{r} \sum_{A_{i}=0}^{D} \psi_{1}^{\mu_{1}} . . \psi_{1}^{\mu_{A_{1}}} \cdots \psi_{r}^{\nu_{1}} . . \psi_{r}^{\nu_{A_{r}}} R_{\mu_{1} . . \mu_{A_{1}}, \cdots, \nu_{1} . . \nu_{A_{r}} ; \alpha}\left|\chi_{\alpha}\right\rangle
$$

where $\left|\chi_{\alpha}\right\rangle$ is a generic state of the fermionic space, $\gamma^{\mu}\left|\chi_{\alpha}\right\rangle=\gamma_{\alpha^{\prime} \alpha}^{\mu}\left|\chi_{\alpha^{\prime}}\right\rangle$, and $a_{r}$ is a numerical prefactor that will be fixed shortly (cfr. eq. (3.5)). In particular we impose a minimal set of constraints on the physical state; the remaining constraints are automatically satisfied thanks to the above algebra. The set of constraints we impose is the following

1. $J_{I}{ }^{J}|R\rangle=0$; for $I=J$, these constraints yield irreducibility conditions and pick-out from the Hilbert space the tensor $R_{\mu_{1} . . \mu_{d}, \cdots, \nu_{1} . . \nu_{d} ; \alpha}$, that is antisymmetric within each block of $d$ of indices and symmetric in the exchange of two blocks. For $I \neq J$, the constraints impose algebraic Bianchi identities $R_{\left[\mu_{1} \ldots \mu_{d}, \nu_{1}\right] . . \nu_{d}, \cdots, \lambda_{1} . . \lambda_{d} ; \alpha}=0$.

2. $Q_{I}|R\rangle=0$; these constraints impose integrability conditions on the physical field. One can thus solve such conditions by writing the "curvature" $|R\rangle$ in terms of a "potential" $|R\rangle=q|\phi\rangle$ where $q$ is a differential operator written in terms of the algebra operators. In terms of the potential the above constraints now become differential Bianchi identities.

3. $L^{I}|R\rangle=0$ : these constraints correspond to "gamma-tracelessness" of the higher-spin curvature $\gamma_{\alpha \alpha^{\prime}}^{\mu} R_{\mu \ldots . \mu_{d}, \cdots, \nu_{1} . . \nu_{d} ; \alpha^{\prime}}=0$.

The remaining constraints are automatically satisfied. The trace constraints $K^{I J}$ are satisfied thanks to the first equation in (2.17); $K_{I J}$ are satisfied because they are trace constraints for the dual curvature obtained using the dual basis for the operators labelled by $I$ and $J$ : it is easy to convince oneself that such dual trace constraints are satisfied if $K^{I J}$ are, because of the relation $\epsilon_{\mu_{1} \cdots \mu_{d}} \epsilon^{\nu_{1} \cdots \nu_{d}}=\delta_{\left.\mu_{1} \cdots \mu_{d}\right]}^{\left[\nu_{1} \cdots \nu_{d}\right]}$. In turn $L_{I}$ are satisfied thanks to the second equation in (2.16), $\not P$ is satisfied thanks to (2.20) and in turn $H$ is satisfied thanks to the second equation in (2.12). Finally $Q^{I}$ are satisfied thanks to the first equation in (2.19). ${ }^{1}$

For $D$ and $N$ arbitrary, the above constraints correspond to the free equations of motion of a conformal particle in flat $D$ dimensions [19]: the algebraic constraints pick out a representation of the conformal group $S O(2, D)$ represented by the rectangular Young tableau with $d=\frac{D}{2}$ rows and $r$ columns.

\footnotetext{
${ }^{1}$ For spin $3 / 2(r=1)$ there are no traces. In this case it is easy to prove that $\gamma^{\mu_{1}} R_{\mu_{1} \cdots \mu_{d}}=$ $0 \Rightarrow \gamma \gamma^{\nu_{2} \cdots \nu_{d} \mu_{1} \cdots \mu_{d}} R_{\mu_{1} \cdots \mu_{d}}=\epsilon^{\nu_{1} \cdots \nu_{d} \mu_{1} \cdots \mu_{d}} \gamma_{\nu_{1}} R_{\mu_{1} \cdots \mu_{d}}=0$. The first term and the last term are respectively $L^{1}|R\rangle=0$ and $L_{1}|R\rangle=0$ expressed in components.
} 


\section{Higher-spin curvature}

In flat space it is easy to solve the integrability conditions since $Q_{I}$ 's anticommute. Hence

$$
|R\rangle=q|\phi\rangle, \quad q=\frac{1}{r !} \epsilon^{I_{1} \cdots I_{r}} Q_{I_{1}} \cdots Q_{I_{r}}
$$

with $J_{I}^{J}|\phi\rangle=-\delta_{I}^{J}|\phi\rangle$ and

$$
|\phi\rangle \sim \psi_{1}^{\mu_{1}} . . \psi_{1}^{\mu_{d-1}} \cdots \psi_{r}^{\nu_{1}} . . \psi_{r}^{\nu_{d-1}} \phi_{\mu_{1} . . \mu_{d-1}, \cdots, \nu_{1} . . \nu_{d-1} ; \alpha}\left|\chi_{\alpha}\right\rangle
$$

In components it reads

$$
R_{\mu_{1} . . \mu_{d}, \cdots, \nu_{1} . . \nu_{d} ; \alpha}=\partial_{\mu_{1}} \cdots \partial_{\nu_{1}} \phi_{\mu_{2} . . \mu_{d}, \cdots, \nu_{2} . . \nu_{d} ; \alpha}
$$

with implied antisymmetrization among each block of indices and symmetrization between exchange of two blocks. Above we fixed

$$
a_{r}= \begin{cases}1, & r \text { even } \\ -i, & r \text { odd }\end{cases}
$$

in order to obtain a real-valued normalization for the curvature.

\section{Higher-derivative equations of motion and their gauge invariance}

The gamma-trace conditions imposed upon the curvature yield the higher-curvature equations of motion satisfied by the associated gauge potential. By rewriting the curvature operator as

$$
q=\frac{1}{r} Q_{J} q^{J}=Q_{1} q^{1}, \quad q^{I} \equiv \frac{1}{(r-1) !} \epsilon^{I I_{2} \cdots I_{r}} Q_{I_{2}} \cdots Q_{I_{r}}
$$

the gamma-trace constraints read

$$
L^{1} q|\phi\rangle=L^{1} Q_{1} q^{1}|\phi\rangle=0
$$

and, using the above algebra, it reduces to

$$
(-)^{r-1} q^{1}\left(\not P+Q_{1} L^{1}\right)|\phi\rangle=(-)^{r-1} q^{1}\left(\not P+Q_{K} L^{K}\right)|\phi\rangle=0
$$

where in the last equality we used the nilpotency condition of the $Q_{I}$ 's, so that

$$
q^{I} \mathcal{F}|\phi\rangle=L^{I} q|\phi\rangle=0
$$

where

$$
\mathcal{F}=(-)^{r-1}\left(\not P+Q_{K} L^{K}\right)
$$


is the Fang-Fronsdal operator [11] that is the higher-spin generalization of the Dirac and Rarita-Schwinger operators and the expression

$$
L^{I}|R\rangle=q^{I} \mathcal{F}|\phi\rangle
$$

is the half-integer higher-spin generalization of the Damour-Deser identity [7].

By defining the gauge transformation

$$
\delta_{\xi}|\phi\rangle=Q_{K} V^{K}|\xi\rangle
$$

where $V^{K}=V^{\mu}(x) \psi_{\mu}^{K}$ and $|\xi\rangle$ being a tensor of the same species as $|\phi\rangle$, we have

$$
\delta_{\xi} \mathcal{F}|\phi\rangle=(-)^{r-1} Q_{I} Q_{J} V^{J} L^{I}|\xi\rangle
$$

that is not trivial provided $r \geq 2(s \geq 5 / 2)$. From (3.13) the gauge invariance of the higher-derivative equations of motion (3.9) immediately follows

$$
\delta_{\xi} q^{I} \mathcal{F}|\phi\rangle=0
$$

thanks again to the nilpotency of $Q_{I}$ 's. Such a property also yields

$$
\mathcal{F}|\phi\rangle=Q_{I} Q_{J} W^{J} W^{I}|\rho\rangle
$$

as generic kernel of $q^{I}$ in (3.9). The latter is gauge-invariant provided the "compensator" field transforms as

$$
\delta_{\xi} W^{J} W^{I}|\rho\rangle=(-)^{r-1} V^{[J} L^{I]}|\xi\rangle
$$

\section{Fang-Fronsdal equation of motion: partial gauge fixing}

The Fang-Fronsdal linear equation of motion

$$
\mathcal{F}|\phi\rangle=0
$$

for a free spin-s field can be obtained from the compensated linear equation (3.15) by gauging away the compensator $V^{[J} L^{I]}|\xi\rangle=-W^{J} W^{I}|\rho\rangle$. This condition, and in turn the Fang-Fronsdal equation, are preserved by a gauge symmetry, given by (3.12) subject to the algebraic constraint

$$
Q_{J} Q_{K} V^{K} L^{J}|\xi\rangle=0
$$

that is non-trivial when $r \geq 2(s \geq 5 / 2)$ and corresponds to gamma-tracelessness of the gauge parameter

$$
L^{I}|\xi\rangle=0 \text {. }
$$


In turn, the gauge potential must satisfy algebraic constraints. After a little algebra one obtains

$$
0=\left(Q_{I} L^{I} \not P+\frac{2}{3} Q_{I} Q_{J} L^{I} L^{J}\right) \mathcal{F}|\phi\rangle=(-)^{r} Q_{I} Q_{J} Q_{K} L^{I} L^{J} L^{K}|\phi\rangle
$$

that is non-trivial when $s \geq 7 / 2$ and corresponds to triple gamma-tracelessness of the gauge potential.

We thus see how neatly the present complex basis of the $S O(N)$-extended spinning particle constraint algebra reproduces all the known features of free higher spin field theory in flat space.

\section{An example: spin- $\frac{7}{2}$ in four dimensions}

To make contact with the standard notation we specialize to the lowest spin that develops all the features described above, spin- $\frac{7}{2}$. In such a case the curvature is given by

$$
|R\rangle=Q_{1} Q_{2} Q_{3}|\phi\rangle \quad \rightarrow \quad R_{\lambda_{1} \lambda_{2} \mu_{1} \mu_{2} \nu_{1} \nu_{2} ; \alpha}=\partial_{\lambda_{1}} \partial_{\mu_{1}} \partial_{\nu_{1}} \phi_{\lambda_{2} \mu_{2} \nu_{2} ; \alpha}
$$

with implied antisymmetrization within pairs of indices (e.g. $\lambda_{1} \leftrightarrow \lambda_{2}$ ) and symmetry between exchange of pairs (e.g. $\lambda_{1} \lambda_{2} \leftrightarrow \mu_{1} \mu_{2}$ ). The cubic equation of motion following from gamma-tracelessness is

$$
\begin{aligned}
& L^{1}|R\rangle=q^{1} \mathcal{F}|\phi\rangle=0 \\
& \rightarrow \partial_{\mu_{1}} \partial_{\nu_{1}}\left(\not \partial \phi_{\lambda_{2} \mu_{2} \nu_{2}}-\partial_{\lambda_{2}} \gamma^{\lambda} \phi_{\lambda \mu_{2} \nu_{2}}-\partial_{\mu_{2}} \gamma^{\mu} \phi_{\lambda_{2} \mu \nu_{2}}-\partial_{\nu_{2}} \gamma^{\nu} \phi_{\lambda_{2} \mu_{2} \nu}\right)=0
\end{aligned}
$$

where spinorial indices have been suppressed and $\phi$ is a totally symmetric tensor; here Damour-Deser identity and Fang-Fronsdal linear operator are self-evident. The latter equation is gauge invariant under the unconstrained gauge symmetry

$$
\delta|\phi\rangle=Q_{K} V^{K}|\xi\rangle \quad \rightarrow \quad \delta \phi_{\lambda_{1} \mu_{1} \nu_{1}}=\partial_{\lambda_{1}} \zeta_{\mu_{1} \nu_{1}}+\partial_{\mu_{1}} \zeta_{\lambda_{1} \nu_{1}}+\partial_{\nu_{1}} \zeta_{\lambda_{1} \mu_{1}}
$$

thanks to the above antisymmetry; here $\zeta_{\mu \nu}=-i V^{\lambda} \xi_{\lambda \mu \nu}$. The cubic equation of motion can be reduced to a compensated linear equation

$$
\begin{aligned}
\mathcal{F}|\phi\rangle & =Q_{I} Q_{J} W^{J} W^{I}|\rho\rangle \\
& \downarrow \\
\not \phi_{\lambda \mu \nu} & -\partial_{\lambda} \gamma^{\lambda^{\prime}} \phi_{\lambda^{\prime} \mu \nu}-\partial_{\mu} \gamma^{\mu^{\prime}} \phi_{\lambda \mu^{\prime} \nu}-\partial_{\nu} \gamma^{\nu^{\prime}} \phi_{\lambda \mu \nu^{\prime}}=-2\left(\partial_{\lambda} \partial_{\mu} \sigma_{\nu}+\partial_{\nu} \partial_{\lambda} \sigma_{\mu}+\partial_{\mu} \partial_{\nu} \sigma_{\lambda}\right)
\end{aligned}
$$

with $\sigma_{\nu}=W^{\lambda} W^{\mu} \rho_{\lambda \mu \nu}$ being the compensator field. The latter is gauge-invariant with unconstrained parameter if the compensator field transforms as $\delta \sigma_{\nu}=\gamma^{\mu^{\prime}} \zeta_{\mu^{\prime} \nu}$. Hence the Fang-Fronsdal equation

$$
\not \partial \phi_{\lambda \mu \nu}-\partial_{\lambda} \gamma^{\lambda^{\prime}} \phi_{\lambda^{\prime} \mu \nu}-\partial_{\mu} \gamma^{\mu^{\prime}} \phi_{\lambda \mu^{\prime} \nu}-\partial_{\nu} \gamma^{\nu^{\prime}} \phi_{\lambda \mu \nu^{\prime}}=0
$$

is consistent provided $\gamma^{\lambda} \gamma^{\mu} \gamma^{\nu} \phi_{\lambda \mu \nu}=0$ (cfr. eq. (3.20)) and it is gauge invariant provided $\gamma^{\mu^{\prime}} \zeta_{\mu^{\prime} \nu}=0$. 


\section{Higher spin equations of motion in (A)dS space}

Similarly to the flat space case discussed in the previous section, geometric equations for higher spins in (A)dS can be obtained by imposing the $S O(2 r+1)$ spinning algebra generators as constraints on the wave function. In the present case, as discussed in Section 2, the algebra is a quadratic deformation of the flat Lie algebra: it is no more a Lie algebra but is still first class. In order to better solve such constraints, we found it convenient to "rotate" the $S O(2 r)$ susy generators

$$
\mathcal{Q}_{I}^{( \pm)}=Q_{I} \pm \frac{\sqrt{b}}{2} L_{I}
$$

that satisfy the anti-commutation relations

$$
\mathcal{Q}_{I}^{(+)} \mathcal{Q}_{J}^{(-)}+\mathcal{Q}_{J}^{(+)} \mathcal{Q}_{I}^{(-)}=b\left(K_{I L} J_{J}^{L}+K_{J L} J_{I}{ }^{L}\right)=\mathcal{Q}_{I}^{(-)} \mathcal{Q}_{J}^{(+)}+\mathcal{Q}_{J}^{(-)} \mathcal{Q}_{I}^{(+)}
$$

and have the same commutation properties with the $S O(2 r)$ generators $\left(J_{I}^{J}, K_{I J}\right.$, $K^{I J}$ ) as had the original susy operators $Q_{I}$.

The set of independent constraints we now impose is:

1. $J_{I}{ }^{J}|R\rangle=0$; irreducibility conditions + algebraic Bianchi identities: it selects the same $G L(D)$ Young tableau as in flat space.

2. $\mathcal{Q}_{I}^{(-)}|R\rangle=0$; integrability conditions: it yields a gauge-invariant curvature.

3. $L^{I}|R\rangle=0$; gamma-tracelessness: it yields higher-curvature equation of motion and Damour-Deser identity.

All other constraints are satisfied thanks to the (A)dS-deformed spinning particle algebra described in Section 2.

\section{Higher-spin curvature}

The explicit expression for the linearized higher-spin curvature in (A)dS, a polynomial in the (A)dS scale $b$, can be obtained by solving the above integrability condition

$$
\mathcal{Q}_{I}^{(-)}|R\rangle=0
$$

and reads

$$
|R\rangle=\sum_{n=0}^{[r / 2]}(-b)^{n} r_{n}(r) q_{n}(r)|\phi\rangle
$$

where the operators $q_{n}(r)$ are now given by (recall that $r=s-1 / 2=[s]$ )

$$
q_{n}(r)=\frac{1}{r !} \epsilon^{I_{1} \cdots I_{r}} \times\left\{\begin{array}{lll}
K_{I_{1} I_{2}} \cdots K_{I_{2 n-1} I_{2 n}} & \mathcal{Q}_{I_{2 n+1}}^{(+)} \mathcal{Q}_{I_{2 n+2}}^{(-)} \cdots \mathcal{Q}_{I_{r}}^{(-)}, & r=2 p \\
K_{I_{1} I_{2}} \cdots K_{I_{2 n-1} I_{2 n}} & \mathcal{Q}_{I_{2 n+1}}^{(+)} \mathcal{Q}_{I_{2 n+2}}^{(-)} \cdots \mathcal{Q}_{I_{r}}^{(+)}, & r=2 p+1
\end{array}\right.
$$


and with $r_{n}(r)$ numerical coefficients recursively given in terms of the Pochhammer function $P(r, k) \equiv r(r-1)(r-2) \cdots(r-k)$ as follows

$$
r_{n}(r)=\frac{1}{2 n} \sum_{k=1}^{n} r_{n-k}(r) a_{2 k}(r-2(n-k)+1), \quad r_{0}(r) \equiv 1
$$

where

$$
a_{2 k}(r)=f_{k} P(r, 2 k)=f_{k} \prod_{l=0}^{2 k}(r-l)
$$

and the $r$-independent coefficients $f_{k}$ are generated by the Taylor expansion of the tangent function, $\tan (z)=\sum_{k=0}^{\infty} f_{k} z^{2 k+1}$.

In the integer spin case [20] the anti-commutation relations of susy generators $Q_{I}$ were precisely the same as (4.2) and, along with the irreducibility conditions $J_{I}{ }^{J}|R\rangle=0$, were the only needed relations to solve the integrability conditions in (A)dS and obtain the higher spin curvatures. Hence, due to the anti-commutation relations (4.2) the above operators $q_{n}(r)$ satisfy the same identities as did their integer spin counterparts (see Appendix B of [20]). Therefore linear combinations of operators $q_{n}(r)$ with the same coefficients $r_{n}(r)$ as in the integer spin case (with the replacement $s \rightarrow r$ ) must satisfy the above Bianchi identity. In summary, the correspondence between the integer case and the present half-integer case amounts to the replacements

\begin{tabular}{l|c|c}
\hline Spin & $s \in \mathbb{N}$ & $s=r+1 / 2, r \in \mathbb{N}$ \\
\hline Operators $q_{n}$ & $\frac{1}{r !} \epsilon^{I_{1} \cdots I_{s}} K_{I_{1} I_{2}} \cdots K_{I_{2 n-1} I_{2 n}} Q_{I_{2 n+1}} \cdots Q_{I_{s}}$ & eq. (4.5) \\
\hline Coefficients & $r_{n}(s)$ & $r_{n}(r)$ \\
\hline Bianchi identity & $Q_{I}|R\rangle=0$ & $\mathcal{Q}_{I}^{(-)}|R\rangle=0$ \\
\hline Gauge transf.'s & $\delta_{\xi}|\phi\rangle=Q_{K} V^{K}|\xi\rangle$ & eq. (4.9) \\
\hline
\end{tabular}

\section{Equations of motion and their gauge invariance}

In the previous section we solved the curvatures in terms of their potential, thus explicitly implementing differential Bianchi identities. Now we can impose gamma 
traces upon the curvatures to get higher-derivative equations of motion

$$
L^{I}|R\rangle=\sum_{n=0}^{[r / 2]}(-b)^{n} r_{n}(r) L^{I} q_{n}(r)|\phi\rangle=0
$$

Gauge transformations of the gauge potentials are given by

$$
\delta_{\xi}|\phi\rangle=\left\{\begin{array}{ll}
\mathcal{Q}_{I}^{(+)} V^{I}|\xi\rangle, & r=2 p \\
\mathcal{Q}_{I}^{(-)} V^{I}|\xi\rangle, & r=2 p+1
\end{array} .\right.
$$

The latter difference may appear bizarre but it is simply due to the fact that the number of $\psi$ 's in the two cases above differ by one. Hence, if we write the transformation in components, when the $\psi$ 's pass to the left of the gamma matrix the sign difference cancels, giving rise to the same gauge transformation as given in [11].

Once again the gamma-trace constraint yields the equation of motion for the field potential

$$
L^{I}|R\rangle=q^{I} \mathcal{F}_{r}|\phi\rangle=0
$$

where $q^{I}$ is the (A)dS counterpart of the operator defined in (3.6) and the $(D$ dimensional generalization of the) Fang-Fronsdal differential operator is expected to be

$$
\begin{aligned}
\mathcal{F}_{r} & =(-)^{r-1}\left(\not I+Q_{K} L^{K}\right)+\frac{\sqrt{b}}{2} L^{K} L_{K} \\
& =(-)^{r-1}\left(\not I+\mathcal{Q}_{K}^{\left(\varepsilon_{r}\right)} L^{K}\right)+\sqrt{b} J_{K}{ }^{K}
\end{aligned}
$$

with $\varepsilon_{r}=(-)^{r-1}$. In fact we note that, using (4.9), the gauge transformation of $\mathcal{F}_{r}|\phi\rangle$ reads

$$
\delta_{\xi} \mathcal{F}_{r}|\phi\rangle=(-)^{r-1}\left(\mathcal{Q}_{[I}^{(-)} \mathcal{Q}_{J]}^{(+)}-b K_{I J}\right) V^{J} L^{I}|\xi\rangle
$$

and is $D$-independent. Morevoer, for $r \geq 2(s \geq 5 / 2)$, it vanishes only if the gauge parameter is gamma-traceless, $L^{I}|\xi\rangle=0$.

\section{Examples}

In the remainder of the section, in order to test our results, we explicitly prove the gauge invariance of the higher spin curvatures and obtain the Damour-Deser identities for the simplest cases,

$r=1,2$. 


\section{(i) Spin $3 / 2$}

With $r=1$ we simply have

$$
|R\rangle=q|\phi\rangle=\mathcal{Q}^{(+)}|\phi\rangle
$$

so that Bianchi identity and gauge invariance

$$
\mathcal{Q}^{(-)} q|\phi\rangle=q \mathcal{Q}^{(-)} V|\xi\rangle=0, \quad \forall \xi
$$

are obvious thanks to the identities $\mathcal{Q}^{(-)} \mathcal{Q}^{(+)}=\mathcal{Q}^{(+)} \mathcal{Q}^{(-)}=0$. The Damour-Deser identity in this case is trivial

$$
L|R\rangle=\mathcal{F}_{1}|\phi\rangle=0
$$

as the gamma-trace of the curvature is linear in derivatives and it is thus identically equal to the Fang-Fronsdal equation of motion.

In order to make contact with conventional notation let us re-write the previous expressions in components: we just need to pull out the fermionic coordinates $\prod \psi^{\mu}$. Equations (4.13-4.14) read

$$
\begin{aligned}
& R_{\mu_{1} \cdots \mu_{d}}=\nabla_{\left[\mu_{1}\right.} \phi_{\left.\mu_{2} \cdots \mu_{d}\right]} \\
& \nabla_{\left[\mu_{1}\right.} \nabla_{\mu_{2}} \phi_{\left.\mu_{3} \cdots \mu_{d+1}\right]}=\nabla_{\left[\mu_{1}\right.} \nabla_{\mu_{2}} \zeta_{\left.\mu_{3} \cdots \mu_{d}\right]}=0
\end{aligned}
$$

where $\nabla_{\mu}=D_{\mu}+i(-)^{d} \frac{\sqrt{b}}{2} \gamma_{\mu}$ is the so-called $(S O(2, D-1)) S O(1, D)$ covariant derivative in $(\mathrm{A}) \mathrm{dS}\left(D_{\mu}\right.$ is the standard covariant derivative in (A)dS) and $\zeta_{\mu_{1} \cdots \mu_{d-2}}=$ $-i V^{\mu} \xi_{\mu \mu_{1} \cdots \mu_{d-2}}$. The commutator of two $\nabla$ 's acts trivially on the spinor index whereas it acts as the standard commutator on space-time indices. Hence, differential Bianchi identity and gauge invariance (cfr. eq. (4.17)) are guaranteed thanks to standard algebraic Bianchi identity obeyed by the (A)dS Riemann tensor. The gauge-invariant Fang-Fronsdal equation (4.15) in components reads

$$
\not D \phi_{\mu_{2} \cdots \mu_{d}}+i(-)^{d} \sqrt{b} \phi_{\mu_{2} \cdots \mu_{d}}-(d-1) \tilde{\nabla}_{\left[\mu_{2}\right.} \gamma \cdot \phi_{\left.\mu_{3} \cdots \mu_{d}\right]}=0
$$

with $\tilde{\nabla}_{\mu}=D_{\mu}-i(-)^{d} \frac{\sqrt{b}}{2} \gamma_{\mu}$. In four dimensions, $d=2$, the previous equations reduce to the known gravitino equations in (A)dS.

(ii) Spin $5 / 2$

This case is the simplest case where most features appear non-trivially. For the curvature, from equations (4.4-4.7), one explicitly obtains

$$
|R\rangle=q|\phi\rangle=\frac{1}{2} \epsilon^{I_{1} I_{2}}\left(\mathcal{Q}_{I_{1}}^{(+)} \mathcal{Q}_{I_{2}}^{(-)}-b K_{I_{1} I_{2}}\right)|\phi\rangle
$$


whose gamma-traceless conditions yield

$$
L^{I}|R\rangle=q^{I} \mathcal{F}_{2}|\phi\rangle=0
$$

with $q^{I}=\epsilon^{I J} \mathcal{Q}_{J}^{(+)}$. Above the first equality is the Damour-Deser identity and the second is the higher-curvature equation of motion for the spin-5/2 in (A)dS. The expression (4.19) is by construction Bianchi-identical with respect to $\mathcal{Q}_{I}^{(-)}$, and it not difficult to check that (4.19) and (4.20) are gauge invariant with respect to (4.9), with unconstrained parameter. In turn, this yields the unconstrained compensated equation of motion

$$
\mathcal{F}_{2}|\phi\rangle=\left(\mathcal{Q}_{I}^{(-)} \mathcal{Q}_{J}^{(+)}-b K_{I J}\right) W^{J} W^{I}|\rho\rangle
$$

as kernel of $q^{I}$, that is gauge-invariant provided

$$
\delta_{\xi} W^{J} W^{I}|\rho\rangle=-V^{[J} L^{I]}|\xi\rangle
$$

that has the same form as its flat counterpart (3.16); the Fang-Fronsdal equation of motion $\mathcal{F}_{2}|\phi\rangle=0$, obtained by gauging away the compensator, is preserved by gamma-traceless gauge transformations.

(iii) Spin $>5 / 2$

The explicit forms of the higher spin curvatures are again given by (4.4-4.7). Although for these cases we do not have an explicit form of the operator $q^{I}$ appearing in the Damour-Deser identity (4.10), we do know the Fang-Fronsdal operators in (A)dS spaces and their gauge transformations (cfr. eq.'s (4.11-4.12)); we can thus infer that equations (4.21) and (3.16) hold unchanged for generic spin in (A)dS.

\section{Conclusions and Outlook}

In the present manuscript (a generalization of [20]) we used locally supersymmetric $O(2 r+1)$-extended spinning particle models in maximally symmetric $D$-dimensional spaces to compute higher spin linearized curvatures for some half-integer spin fields (those corresponding to Young tableaux with $\frac{D}{2}-1$ rows and $r$ columns) and we used them to analyze higher spin equations of motion for some specific cases. Equations (4.4-4.7) are the main results of the manuscript.

The $O(N)$-extended spinning particles are easily seen to be Weyl invariant for all $N$ : using this property in [20] it was shown how they consistently propagate in generic conformally flat spaces. In fact the associated constraint algebra keeps being first class, though in a non-linear way. This might indicate that the present analysis might have an extension to conformally flat spaces. However, although BRST lagrangian constructions for propagation of (massive) spin-3/2 field and spin-2 
in spaces more generic than maximally symmetric ones were recently constructed [31], for spin larger than two, the higher spin counterparts of such BRST algebras appear to close only for maximally symmetric spaces. So, even if one were able to solve the above conformally flat spinning particle algebra in terms of a higher-derivative equation of motion for the higher spin potential, it might be problematic to cast the solution into a compensated first-order (second-order for integer spins) equation of motion: it would be very interesting to further clarify this point.

Finally, it would also be quite interesting to find connections between the present particle models and the geometric approach to massive higher-spin theories [32]; in particular, by dimensionally reducing the results obtained in [20] and here, it seems natural to link $O(N)$-extended particle models to massive higher-spin theories [33] defined in odd-dimensional spaces [34].

\section{Acknowledgments}

This work was partly supported by the Italian MIUR-PRIN contract 20075ATT78. The author would like to thank F. Bastianelli for help, discussions and careful reading of the manuscript and E. Latini for discussions.

\section{References}

[1] M. A. Vasiliev, Fortsch. Phys. 52 (2004) 702, [arXiv:hep-th/0401177]; D. Sorokin, AIP Conf. Proc. 767 (2005) 172, [arXiv:hep-th/0405069]; N. Bouatta, G. Compere and A. Sagnotti, "An Introduction to Free Higher-Spin Fields,"

arXiv:hep-th/0409068; X. Bekaert, S. Cnockaert, C. Iazeolla and M. A. Vasiliev, "Nonlinear higher spin theories in various dimensions," arXiv:hep-th/0503128;

A. Fotopoulos and M. Tsulaia, Int. J. Mod. Phys. A 24 (2009) 1 [arXiv:0805.1346 [hep-th]]; P. Benincasa and F. Cachazo, "Consistency Conditions on the S-Matrix of Massless Particles," arXiv:0705.4305 [hep-th]; M. Porrati, Phys. Rev. D 78 (2008) 065016 [arXiv:0804.4672 [hep-th]].

[2] C. Schubert, Phys. Rept. 355 (2001) 73 [arXiv:hep-th/0101036].

[3] V. D. Gershun and V. I. Tkach, Pisma Zh. Eksp. Teor. Fiz. 29 (1979) 320 [Sov. Phys. JETP 29 (1979) 288].

[4] P. S. Howe, S. Penati, M. Pernici and P. K. Townsend, Phys. Lett. B 215 (1988) 555; Class. Quant. Grav. 6 (1989) 1125.

[5] D. Francia and A. Sagnotti, Phys. Lett. B 543 (2002) 303 [arXiv:hep-th/0207002]; Class. Quant. Grav. 20 (2003) S473 [arXiv:hep-th/0212185]; Phys. Lett. B 624 (2005) 93 [arXiv:hep-th/0507144]; D. Francia, J. Mourad and A. Sagnotti, Nucl. Phys. B 773 (2007) 203 [arXiv:hep-th/0701163]; Nucl. Phys. B 804 (2008) 383 
[arXiv:0803.3832 [hep-th]]; see D. Francia, J. Phys. Conf. Ser. 222 (2010) 012002 [arXiv:1001.3854 [hep-th]] for a recent report and an updated list of references.

[6] S. Weinberg, Phys. Rev. 138 (1965) B988; B. de Wit and D. Z. Freedman, Phys. Rev. D 21 (1980) 358.

[7] T. Damour and S. Deser, Annales Poincare Phys. Theor. 47 (1987) 277.

[8] M. Dubois-Violette and M. Henneaux, Lett. Math. Phys. 49 (1999) 245 [arXiv:math/9907135]; Commun. Math. Phys. 226 (2002) 393 [arXiv:math/0110088].

[9] C. Fronsdal, Phys. Rev. D 18 (1978) 3624; Phys. Rev. D 20 (1979) 848.

[10] J. M. F. Labastida and T. R. Morris, Phys. Lett. B 180 (1986) 101;

J. M. F. Labastida, Phys. Rev. Lett. 58 (1987) 531; Nucl. Phys. B 322 (1989) 185; see also, S. Ouvry and J. Stern, Phys. Lett. B 177 (1986) 335; W. Siegel and B. Zwiebach, Nucl. Phys. B 282 (1987) 125.

[11] J. Fang and C. Fronsdal, Phys. Rev. D 18 (1978) 3630.

[12] T. Curtright, Phys. Lett. B 85 (1979) 219.

[13] J. Fang and C. Fronsdal, Phys. Rev. D 22 (1980) 1361.

[14] R. Marnelius, "Lagrangian higher spin field theories from the $\mathrm{O}(\mathrm{N})$ extended supersymmetric particle," arXiv:0906.2084 [hep-th].

[15] F. Bastianelli, O. Corradini and E. Latini, JHEP 0702 (2007) 072 [arXiv:hep-th/0701055].

[16] F. Bastianelli and A. Zirotti, Nucl. Phys. B 642 (2002) 372 [arXiv:hep-th/0205182];

F. Bastianelli, O. Corradini and A. Zirotti, Phys. Rev. D 67 (2003) 104009 [arXiv:hep-th/0211134]; JHEP 0401 (2004) 023 [arXiv:hep-th/0312064];.

F. Bastianelli, P. Benincasa and S. Giombi, JHEP 0504 (2005) 010 [arXiv:hep-th/0503155]; JHEP 0510 (2005) 114 [arXiv:hep-th/0510010].

[17] S. M. Kuzenko and Z. V. Yarevskaya, Mod. Phys. Lett. A 11 (1996) 1653 [arXiv:hep-th/9512115].

[18] R. Marnelius, Phys. Rev. D 20 (1979) 2091.

[19] W. Siegel, Int. J. Mod. Phys. A 3 (1988) 2713; Int. J. Mod. Phys. A 4 (1989) 2015; R. R. Metsaev, Mod. Phys. Lett. A 10 (1995) 1719; see also, X. Bekaert and M. Grigoriev, SIGMA 6 (2010) 038 [arXiv:0907.3195 [hep-th]].

[20] F. Bastianelli, O. Corradini and E. Latini, JHEP 0811 (2008) 054 [arXiv:0810.0188 [hep-th]].

[21] R. Manvelyan and W. Ruhl, Nucl. Phys. B 797 (2008) 371 [arXiv:0705.3528 [hep-th]]; Nucl. Phys. B 796 (2008) 457 [arXiv:0710.0952 [hep-th]]; J. Engquist and O. Hohm, JHEP 0804 (2008) 101 [arXiv:0708.1391 [hep-th]]. 
[22] I. A. Bandos and J. Lukierski, Mod. Phys. Lett. A 14 (1999) 1257

[arXiv:hep-th/9811022]; I. A. Bandos, J. Lukierski and D. P. Sorokin, Phys. Rev. D 61 (2000) 045002 [arXiv:hep-th/9904109]; I. Bandos, X. Bekaert, J. A. de Azcarraga, D. Sorokin and M. Tsulaia, JHEP 0505 (2005) 031 [arXiv:hep-th/0501113].

[23] F. Bastianelli and R. Bonezzi, JHEP 0903 (2009) 063 [arXiv:0901.2311 [hep-th]]; F. Bastianelli and R. Bonezzi, JHEP 1005 (2010) 020 [arXiv:1003.1046 [hep-th]].

[24] F. Bastianelli, O. Corradini and A. Waldron, JHEP 0905 (2009) 017 [arXiv:0902.0530 [hep-th]]; symplectic algebras in the study of mixed-symmetry higher spin field theory also appeared in, M. A. Vasiliev, Phys. Rev. D 66 (2002) 066006 [arXiv:hep-th/0106149]; K. B. Alkalaev, M. Grigoriev and I. Y. Tipunin, Nucl. Phys. B 823 (2009) 509 [arXiv:0811.3999 [hep-th]]; K. B. Alkalaev and M. Grigoriev, Nucl. Phys. B 835 (2010) 197 [arXiv:0910.2690 [hep-th]].

[25] K. Hallowell and A. Waldron, Commun. Math. Phys. 278 (2008) 775 [arXiv:hep-th/0702033].

[26] D. Cherney, E. Latini and A. Waldron, J. Math. Phys. 51 (2010) 062302 [arXiv:0906.4814 [hep-th]]; Phys. Lett. B 682 (2010) 472 [arXiv:0909.4578 [hep-th]].

[27] X. Bekaert and N. Boulanger, Commun. Math. Phys. 245 (2004) 27 [arXiv:hep-th/0208058]; Phys. Lett. B 561 (2003) 183 [arXiv:hep-th/0301243]; Yu. M. Zinoviev, "On massive mixed symmetry tensor fields in Minkowski space and (A)dS," arXiv:hep-th/0211233; "First order formalism for mixed symmetry tensor fields," arXiv:hep-th/0304067; P. de Medeiros and C. Hull, Commun. Math. Phys. 235 (2003) 255 [arXiv:hep-th/0208155]; JHEP 0305 (2003) 019 [arXiv:hep-th/0303036]; K. B. Alkalaev, O. V. Shaynkman and M. A. Vasiliev, Nucl. Phys. B 692 (2004) 363 [arXiv:hep-th/0311164]; K. B. Alkalaev, Theor. Math. Phys. 140 (2004) 1253 [Teor. Mat. Fiz. 140 (2004) 424] [arXiv:hep-th/0311212].

A. Campoleoni, D. Francia, J. Mourad and A. Sagnotti, Nucl. Phys. B 815 (2009) 289 [arXiv:0810.4350 [hep-th]]; Nucl. Phys. B 828 (2010) 405 [arXiv:0904.4447 [hep-th]]; A. Campoleoni, Riv. Nuovo Cim. 033 (2010) 123 [arXiv:0910.3155 [hep-th]].

[28] I. L. Buchbinder, A. Pashnev and M. Tsulaia, Phys. Lett. B 523 (2001) 338 [arXiv:hep-th/0109067]; A. Sagnotti and M. Tsulaia, Nucl. Phys. B 682 (2004) 83 [arXiv:hep-th/0311257]; I. L. Buchbinder, V. A. Krykhtin and A. A. Reshetnyak, Nucl. Phys. B 787 (2007) 211 [arXiv:hep-th/0703049]; in G. Bonelli, JHEP 0311 (2003) 028 [arXiv:hep-th/0309222], higher spin constraint algebras in AdS(D) were studied by directly working in the $\mathrm{R}(\mathrm{D}+1)$ hyperboloid.

[29] V. Bargmann and E. P. Wigner, Proc. Nat. Acad. Sci. 34 (1948) 211.

[30] R. Marnelius and U. Martensson, Nucl. Phys. B 321 (1989) 185; W. Siegel, "Fields," chapter XII, arXiv:hep-th/9912205. 
[31] I. L. Buchbinder and V. A. Krykhtin, Mod. Phys. Lett. A 25 (2010) 1667 [arXiv:1003.0185 [hep-th]]; I. L. Buchbinder, V. A. Krykhtin and P. M. Lavrov, Phys. Lett. B 685 (2010) 208 [arXiv:0912.0611 [Unknown]].

[32] D. Francia, Nucl. Phys. B 796 (2008) 77 [arXiv:0710.5378 [hep-th]].

[33] S. Deser and A. Waldron, Phys. Rev. Lett. 87 (2001) 031601 [arXiv:hep-th/0102166]; Yu. M. Zinoviev, "On massive high spin particles in (A)dS," arXiv:hep-th/0108192; P. de Medeiros, Class. Quant. Grav. 21 (2004) 2571 [arXiv:hep-th/0311254]; R. R. Metsaev, Phys. Lett. B 590 (2004) 95 [arXiv:hep-th/0312297]; Class. Quant. Grav. 22 (2005) 2777 [arXiv:hep-th/0412311]; I. L. Buchbinder and V. A. Krykhtin, Nucl. Phys. B 727 (2005) 537 [arXiv:hep-th/0505092]; I. L. Buchbinder, V. A. Krykhtin, L. L. Ryskina and H. Takata, Phys. Lett. B 641 (2006) 386 [arXiv:hep-th/0603212]; I. L. Buchbinder and A. V. Galajinsky, JHEP 0811 (2008) 081 [arXiv:0810.2852 [hep-th]]; E. A. Bergshoeff, O. Hohm and P. K. Townsend, Annals Phys. 325 (2010) 1118 [arXiv:0911.3061 [hep-th]].

[34] Some particle models for odd-dimensional higher spin fields were already proposed some time ago by M. S. Plyushchay, Phys. Lett. B 273 (1991) 250; Int. J. Mod. Phys. A 7 (1992) 7045. 